\title{
Delayed Diagnosis of Small Cell Lung Cancer in a Patient with Allergic Bronchopulmonary Aspergillosis
}

\author{
Won Sik Jung ${ }^{1}$, Jin Won Jang ${ }^{1}$, Seung Yong Park ${ }^{1,2}$, Yeong Hun Choe ${ }^{1,2}$, Yong Chul Lee ${ }^{1,2, *}$, So Ri Kim ${ }^{1,2, *}$ \\ 'Department of Internal Medicine and Research Center for Pulmonary Disorders, Chonbuk National University Medical School; ${ }^{2}$ Research Institute of Clinical \\ Medicine of Chonbuk National University-Biomedical Research Institute of Chonbuk National University Hospital, Jeonju, Korea
}

\begin{abstract}
Allergic bronchopulmonary aspergillosis (ABPA) is a hypersensitivity disorder induced by Aspergillus fumigatus that occurs generally in patients with underlying chronic airway disorders. However, the development of ABPA associated with lung malignancy remains unknown. An 80-year-old woman was admitted for evaluation of chronic cough. Her radiologic finding was a mass like opacity with mucoid impaction, showing finger-in-glove sign, one of the typical radiologic findings for ABPA. In addition, laboratory tests revealed that her data satisfied the diagnostic criteria for ABPA. Interestingly, final pathologic examination showed that the mass lesion was small cell lung cancer. Here, we describe a case of delayed diagnosis of small cell lung cancer with ABPA, suggesting that a possibility of accompanying lung malignancy such as small cell lung cancer should be considered in patients with ABPA. This case let us realize the importance of clinical suspicion that different entities of diseases can be occurred simultaneously for the correct diagnosis without delay.
\end{abstract}

Keywords: Allergic bronchopulmonary aspergillosis; Small cell lung carcinoma; Delayed diagnosis

\section{INTRODUCTION}

Allergic bronchopulmonary aspergillosis (ABPA) is hypersensitivity reaction to Aspergillus fumigatus colonized in the tracheobronchial tree [1]. It is well known to occur frequently in patients with chronic lung disease like bronchial asthma or cystic fibrosis [2]. Generally, the diagnosis of ABPA is based on the criteria composed of clinical, radiological, and immunological features. Particularly, typical radiologic appearance of ABPA such as bronchiectasis and finger-in-glove sign is useful for the diagnosis of ABPA. However, radiologic findings by itself have limited value for establishing diagnosis especially when clinical presentation of ABPA is vague, mimicking other respiratory disorders [3]. Herein, we report a delayed diagnosis of small cell lung cancer (SCLC) with ABPA whose radiologic findings were more preferred to diagnose ABPA not lung malignancy.

\section{CASE REPORT}

An 80-year-old woman visited Chonbuk National University Hospital clinic to examine recurrent cough that lasted for several months. She had been an asthmatic for 9 years and never-smoked. She had no history of other medical illness. High resolution computed tomography (HRCT) showed central bronchiectasis and bronchial wall thickening with internal mucus retention in right middle, both lower, and left upper lobes of the lung. In addition, there was a mass lesion showing finger-in-glove sign in the posterior segment of the right upper lobe (Fig. $1 \mathrm{~A}, \mathrm{C}$ ), which is compatible radiologic features for ABPA. By satisfaction of clinical and immunological diagnostic criteria, diagnosis of ABPA was established by a history of asthma, immediate skin test reactivity to Aspergillus antigen (skin prick test to $A$. fumigatus: $2 \times 2 \mathrm{~cm}$, histamine: $4 \times 4 \mathrm{~cm}$; intradermal test to $A$. fumigatus: $8 \times 10 \mathrm{~cm}$, histamine: $11 \times 9 \mathrm{~cm} / 13 \times 18 \mathrm{~cm})$, peripheral blood eosinophilia (530/ $\mathrm{mm}^{3}$ ), lung infiltration on chest X-ray or chest computed tomogra-
Correspondence to: So Ri Kim

Department of Internal Medicine, Chonbuk National University Medical School, 567 Baekje-daero, Deokjin-gu, Jeonju 54896, Korea Tel: +82-63-250-2475, Fax: +82-63-259-3212, E-mail: sori@jbnu.ac.kr

*These authors contributed equally to this work as a senior author. Received: Oct. 22, 2015/ Accepted after revision: Nov. 11, 2015
(C) 2015 Soonchunhyang Medical Research Institute This is an Open Access article distributed under the terms of the Creative Commons Attribution Non-Commercial License (http://creativecommons.org/licenses/by-nc/3.0/). 
Jung WS, et al. • Delayed Diagnosis of Small Cell Lung Cancer in a Patient with Allergic Bronchopulmonary Aspergillosis
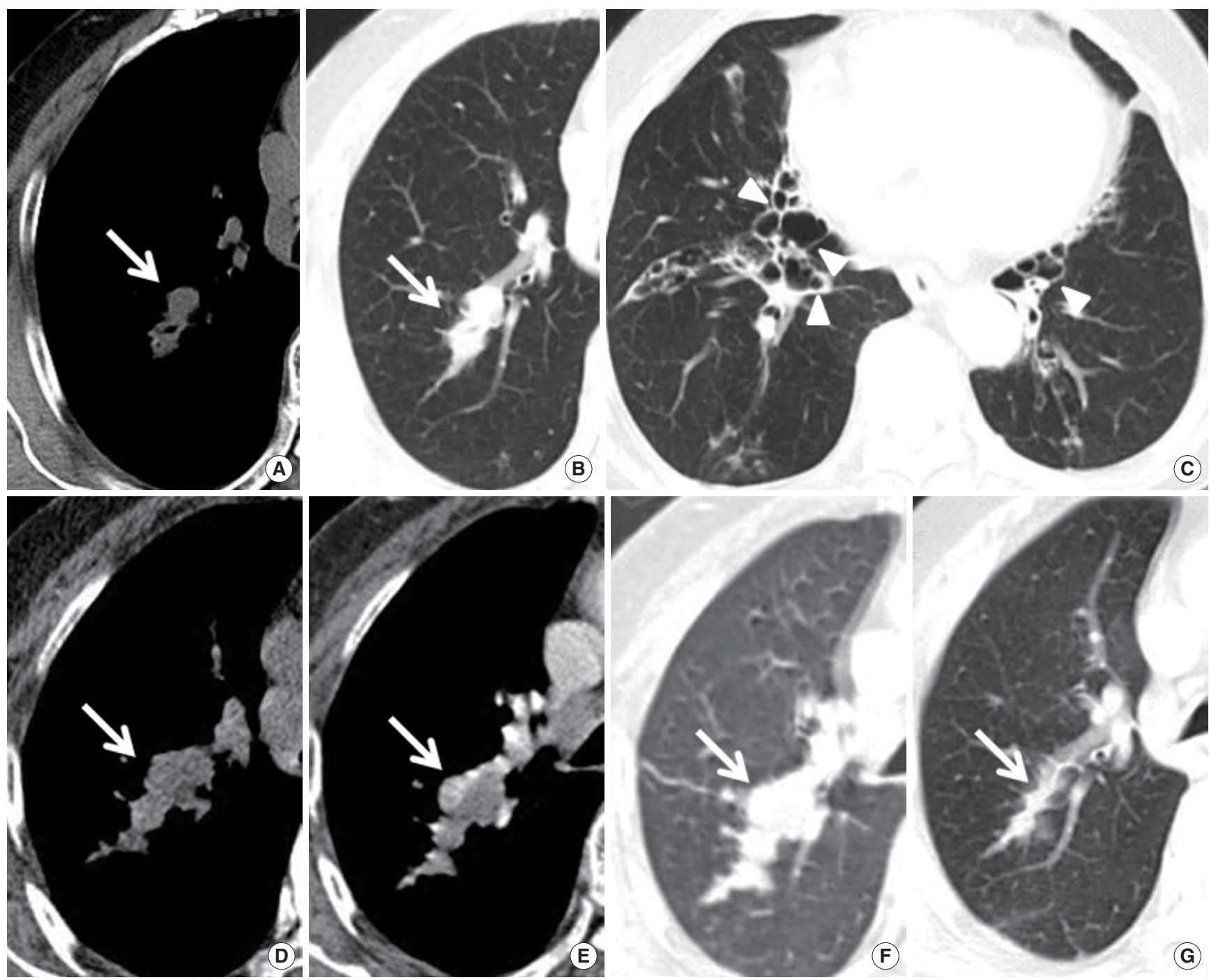

Fig. 1. (A-C) HRCT at admission (arrow and arrowhead). (D-F) Despite 2 months of ABPA treatment with steroid combined itraconazole, follow-up chest computed tomography shows increased size of mass (arrow). (G) Follow-up HRCT shows markedly decreased size (more than 30\%) of mass (arrow). HRCT, high resolution computed tomography.

phy (CT), and central bronchiectasis on chest CT. Oral steroid and itraconazole were started for ABPA treatment. After treatment for 2 months, she complained febrile sensation and chills. Despite 2 months of ABPA treatment with steroid combined itraconazole, follow-up chest CT shows that the lesion is increased in size with lobulated contour nodular opacity that obstructs right upper lobe posterior segmental bronchus. Chest CT revealed mild enhancement pattern and increased size of nodular opacity in the lesion with finger-in-glove sign (Fig. 1D-F). Percutaneous transthoracic needle biopsy was performed, and microscopic examination of the tissue samples revealed that there were round to fusiform shaped cells with scant cytoplasm, fine granular chromatin, and mitotic figure (Fig. 2A, B). On immunohistochemistry for synaptophysin (Fig. 2C), chromogranin, and CD56, the cells were positive. These findings are typical features of SCLC. As for staging work-up, there was a metastatic right lower paratracheal lymphadenopathy on positron emission tomography (PET)-CT, diagnosing limited stage of SCLC. Subsequently, conventional chemotherapy with radiation therapy was introduced to treat SCLC using combination of etoposide and cisplatin (EP). Follow-up HRCT (Fig. 1G) showed markedly decreased size (more than 30\%) of the mass lesion after the first chemotherapy. She had taken 6 cycles of chemotherapy of EP regimen for SCLC. Follow-up HRCT showed stable disease state. Then, she refused the curative or palliative treatment for SCLC. 

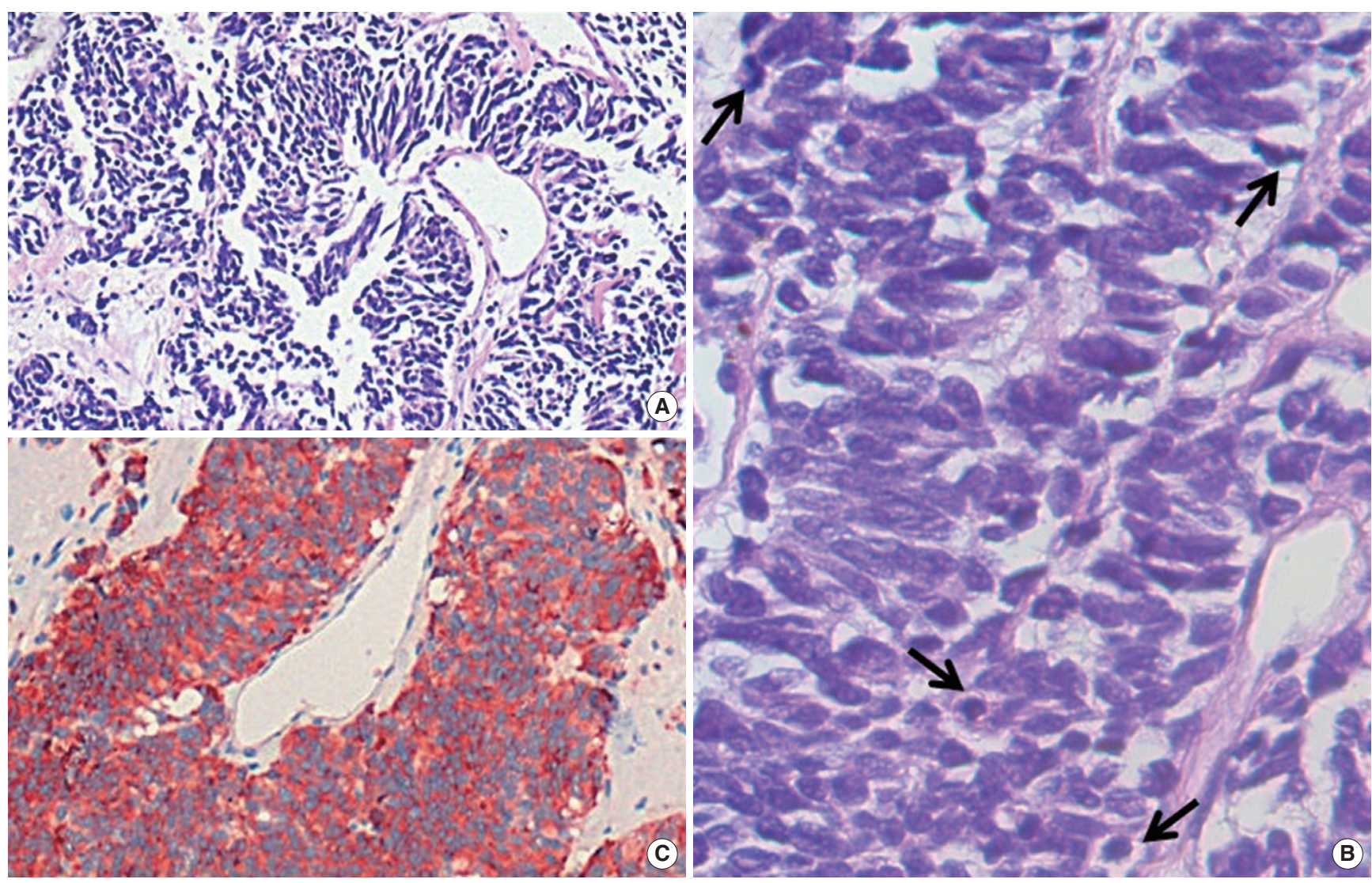

Fig. 2. (A, B) Percutaneous transthoracic needle biopsy reveals that there are nests and cords of round to polygonal cells (black arrows) with scant cytoplasm, granular chromatin, and inconspicuous nucleoli in H\&E stain. (C) Positive stains for synaptophysin by immunohistochemistry was compatible for small cell lung cancer (A and C, $\times 100$ and $B, \times 400)$.

\section{DISCUSSION}

The diagnosis of ABPA is based on clinical, laboratory, and radiologic criteria. Primary criteria include asthma, radiologic evidence of pulmonary infiltration, positive skin test to $A$. fumigatus, eosinophilia, precipitating antibody to A. fumigatus, elevated immunoglobulin E (IgE), elevated A. fumigatus-specific IgE and immunoglobulin $\mathrm{G}$, and central bronchiectasis. A diagnosis of ABPA is nearly certain when six of these eight criteria are fulfilled. However, the confirmative diagnosis of ABPA is still difficult and so controversy in clinical practice.

In fact, several cases of ABPA mimicking lung cancer have been reported [3-5]. However, the simultaneous occurrence of ABPA with other respiratory disorders presenting mass lesion, i.e., lung malignancy is very rare.

On the other hand, similar to our case, 77-year-old male with non-small cell lung cancer (NSCLC) coexisting with ABPA was reported [6]. SCLC coexisting with ABPA has been not reported up to now. The clinical features of ABPA and SCLC are different. So it was more difficult to predict than NSCLC.

Typical radiologic features are helpful for the diagnosis of ABPA. In this case, her radiologic finding was a mass-like opacity with mucoid impaction showing finger-in-glove appearance, one of typical radiologic features of ABPA. In addition, her data of laboratory tests satisfied the diagnostic criteria for ABPA. These include fleeting pulmonary alveolar opacities, mucus plugging with bronchial wall thickening, and central saccular bronchiectasis [7]. Especially, bronchi are plugged with mucus, hyphae, and cellular debris, making up a typical finger-in-glove appearance and it often looks like a mass lesion of the lung. However, unexpectedly, the final pathologic examination revealed that the mass lesion is SCLC and we could know that it is difficult to distinguish ABPA from other respiratory disorders presenting as a mass like lesion just only by radiologic findings.

In this case, she had a history of asthma, immediate skin test reactivity to Aspergillus antigen, peripheral blood eosinophilia, and 
Jung WS, et al. - Delayed Diagnosis of Small Cell Lung Cancer in a Patient with Allergic Bronchopulmonary Aspergillosis

radiologic typical finding of $\mathrm{ABPA}$. These various factors were suggested for ABPA and it did not assess the possibility of other diseases.

We can't explain exactly the cause and effect relationship between lung cancer and ABPA. Double primary possibility can't be ruled out. Rather important point is that although ABPA can be diagnosed clinically, we should think about the possibility of a variety of diseases and additional imaging test (PET-CT) and biopsy may be need for mass lesion.

In conclusion, this case report introduces delayed diagnosis of SCLC with ABPA, suggesting that despite its scarcity the possibility of development ABPA with lung malignancy should be considered. Additionally, this case let us realize the importance of clinical suspicion that different entities of diseases can be occurred simultaneously for the correct diagnosis without delay.

\section{ACKNOWLEDGMENTS}

We thank Professor Mie-Jae Im for critical readings of the manuscript.

\section{REFERENCES}

1. Tillie-Leblond I, Tonnel AB. Allergic bronchopulmonary aspergillosis. Allergy 2005;60:1004-13.

2. Greenberger PA. Allergic bronchopulmonary aspergillosis. J Allergy Clin Immunol 2002;110:685-92.

3. Sanchez-Alarcos JM, Martinez-Cruz R, Ortega L, Calle M, RodriguezHermosa JL, Alvarez-Sala JL. ABPA mimicking bronchogenic cancer. Allergy 2001;56:80-1.

4. Nowicka U, Wiatr E, Jakubowska L, Polubiec-Kownacka M. Allergic bronchopulmonary aspergillosis mimicking lung cancer in a non-asthmatic female patient: a case report. Pol Pneumonol Allergol 2012;80:77-81.

5. Aquino SL, Kee ST, Warnock ML, Gamsu G. Pulmonary aspergillosis: imaging findings with pathologic correlation. AJR Am J Roentgenol 1994; 163:811-5.

6. Itano H, Andou A, Date H, Shimizu N. Non-small cell lung cancer coexisting with pulmonary aspergilloma. Jpn J Thorac Cardiovasc Surg 2005; 53:513-6.

7. Thompson BH, Stanford W, Galvin JR, Kurihara Y. Varied radiologic appearances of pulmonary aspergillosis. Radiographics 1995;15:1273-84. 